JOURNAL OF THE

CHUNGCHEONG MATHEMATICAL SOCIETY

Volume 27, No. 1, February 2014

http://dx.doi.org/10.14403/jcms.2014.27.1.39

\title{
SECANT VARIETIES TO THE VARIETY OF REDUCIBLE FORMS
}

\author{
YONG-SU SHIN*
}

\begin{abstract}
We completely classify the dimension of secant varieties $\operatorname{Sec}_{1}\left(\mathbb{X}_{\lambda, 2}\right)$ to the variety of reducible forms in $\mathbb{k}\left[x_{0}, x_{1}, x_{2}\right]$ when $\lambda=(1, \ldots, 1,3, \ldots, 3)$, and also show that they are all nondefective.
\end{abstract}

\section{Introduction}

Let $R=\mathbb{k}\left[x_{0}, x_{1}, \ldots, x_{n}\right]$ be an $(n+1)$-variable polynomial ring over a field $\mathbb{k}$ and let $I$ be a homogeneous ideal of $R$ (or the ideal of a subscheme in $\left.\mathbb{P}^{n}\right)$. Then the numerical function

$$
\mathbf{H}(R / I, t):=\operatorname{dim}_{\mathbb{k}} R_{t}-\operatorname{dim}_{\mathbb{k}} I_{t}
$$

is called a Hilbert function of the ring $R / I$. If $I:=I_{\mathbb{X}}$ is the ideal of a subscheme $\mathbb{X}$ in $\mathbb{P}^{n}$, then we denote the Hilbert function of $\mathbb{X}$ by

$$
\mathbf{H}_{\mathbb{X}}(t):=\mathbf{H}\left(R / I_{\mathbb{X}}, t\right) .
$$

To introduce a star-configuration, we start with varieties of some specific ideals of $R$. In [2], the authors proved that if $F_{1}, \ldots, F_{s}$ are general forms in $R=\mathbb{k}\left[x_{0}, x_{1}, \ldots, x_{n}\right]$ and

$$
\tilde{F}_{j}=\frac{\prod_{i=1}^{s} F_{i}}{F_{j}} \text { for } j=1, \ldots, s,
$$

then

The variety $\mathbb{X}$ in $\mathbb{P}^{n}$ of the ideal

$$
\left(\tilde{F}_{1}, \ldots, \tilde{F}_{s}\right)=\bigcap_{1 \leq i<j \leq s}\left(F_{i}, F_{j}\right) .
$$

$$
\left(\tilde{F}_{1}, \ldots, \tilde{F}_{s}\right)=\bigcap_{1 \leq i<j \leq s}\left(F_{i}, F_{j}\right)
$$

Received September 27, 2013; Accepted January 06, 2014.

2010 Mathematics Subject Classification: Primary 13A02; Secondary 16W50.

Key words and phrases: star-configurations, linear star-configurations, secant varieties.

This research was supported by a grant from Sungshin Women's University in 2013 . 
is called a star-configuration in $\mathbb{P}^{n}$ of type $s$ defined by general forms $F_{1} \ldots, F_{s}$. Furthermore, if $F_{1}, \ldots, F_{s}$ are all general linear forms, then $\mathbb{X}$ is called a linear star-configuration of type $s$ (see also $[1,2,6,7]$ ).

In this paper, we discuss some applications of star-configurations in $\mathbb{P}^{n}$. In other words, we study some examples of secant varieties to the variety of reducible forms in $\mathbb{P}^{2}$, which is not defective, using the sum of ideals of two star-configurations in $\mathbb{P}^{2}$.

In Section 2, we discuss the Hilbert function of the ideal of the union of two star-configurations $\mathbb{X}$ and $\mathbb{Y}$ in $\mathbb{P}^{2}$ when $\lambda=(1, \ldots, 1,3, \ldots, 3)$, which we will use to find the dimension of secant varieties to the variety of reducible forms in Section 3 (see also $[3,4,5]$ ).

In Section 3, we prove that if $\lambda=(1, \ldots, 1,3, \ldots, 3)$, then the secant variety $\operatorname{Sec}_{1}\left(\mathbb{X}_{\lambda, 2}\right)$ to the variety $\mathbb{X}_{\lambda, 2}$ is not defective for $3<d$ (see Theorem 3.5). Finally, we give a question on secant varieties for the further study.

\section{The union of two star-configurations in $\mathbb{P}^{2}$ defined by linear forms and cubic forms}

In this section, we study the Hilbert function and the minimal generators of the ideal the union of two star-configurations in $\mathbb{P}^{2}$, and we use these in the next section. Throughout this paper,

a solid line

a dashed line

$\mathbb{L}_{i} \quad$ is a line defined by a linear form $L_{i}$,

a thick line

$\mathbb{M}_{i}$ is a line defined by a linear form $M_{i}$,

$\mathbb{L}_{i}$ is a line defined by a cubic form $L_{i}^{3}$,

for $1 \leq i \leq s$ with $s \geq 2$. Moreover, we define that

$P_{i, j}$ is a point defined by linear forms $L_{i}, L_{j}$,

$\boldsymbol{P}_{i, j}$ is a double point defined by a linear form and a quadratic form $L_{i}, L_{j}^{2}$,

$\mathcal{P}_{i, j}$ is a triple point defined by a linear form and a cubic form $L_{i}, L_{j}^{3}$,

$Q_{i, j}$ is a point defined by linear forms $M_{i}, M_{j}$, and

$\boldsymbol{Q}_{i, j}$ is a double point defined by a linear form and a quadratic form $M_{i}, M_{j}^{2}$,

where $L_{i}, L_{j}$ and $M_{i}, M_{j}$ are linear forms in $R$ with $i<j$.

Let $\lambda=\left(d_{1}, \ldots, d_{s}\right)$, where $1 \leq d_{1} \leq \cdots \leq d_{s}$ and $d:=\sum_{i=1}^{s} d_{i}$. We denote by $\mathbb{X}^{(\lambda)}$ a star-configuration in $\mathbb{P}^{2}$ defined by forms $F_{1}, \ldots, F_{s}$ in $R=\mathbb{k}\left[x_{0}, x_{1}, x_{2}\right]$ with $\operatorname{deg}\left(F_{i}\right)=d_{i}$ for every $i$. 
Lemma 2.1. Let $\lambda=(1, \ldots, 1,3)$, and $\mathbb{X}_{1}:=\mathbb{X}_{1}^{(\lambda)}$ and $\mathbb{X}_{2}:=\mathbb{X}_{2}^{(\lambda)}$ be star-configurations in $\mathbb{P}^{2}$ with $5 \leq d \leq 7$. Then $\mathbb{X}:=\mathbb{X}_{1} \cup \mathbb{X}_{2}$ has generic Hilbert function. In particular, $\operatorname{dim}_{\mathbb{k}}\left(I_{\mathbb{X}_{1}}+I_{\mathbb{X}_{2}}\right)_{d}=\left(\begin{array}{c}d+2 \\ 2\end{array}\right)$.

Proof. First, we assume that $\mathbb{X}_{1}$ and $\mathbb{X}_{2}$ are defined by $L_{1}, L_{2}, L_{3}^{3}$, and $M_{1}, M_{2}, M_{3} M_{4} M_{5}$, respectively, where $L_{i}$ and $M_{i}$ are linear forms in $R$ for every $i$ (see Figure 1). Furthermore, we assume that $L_{1}$ vanishes on four points in $\mathbb{X}_{1}$, and one more point in $\mathbb{X}_{2}$, defined by two linear forms $M_{1}$ and $M_{2}$, and $L_{2}$ vanishes on three points in $\mathbb{X}_{1}$ and one more point in $\mathbb{X}_{2}$ defined by linear forms $M_{1}$ and $M_{5}$ (see Figure 1 again).

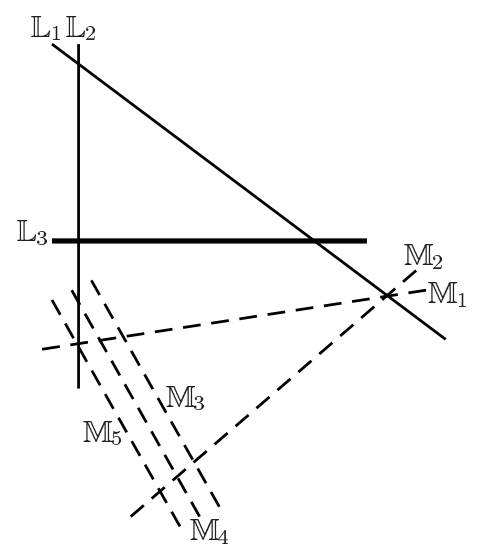

FiguRE 1

By Bezóut's Theorem, for $N \in\left(I_{\mathbb{X}}\right)_{4}, N=\alpha L_{1} L_{2} M_{2} M_{1}$ for some $\alpha \in \mathbb{k}$. Therefore, the Hilbert function of $\mathbb{X}$ is $1 \quad 3 \quad 6 \quad 10 \quad 14 \rightarrow$, as we wished.

Using the following exact sequence

$$
0 \rightarrow R / I_{\mathbb{X}} \rightarrow R / I_{\mathbb{X}_{1}} \oplus R / I_{\mathbb{X}_{2}} \rightarrow R /\left(I_{\mathbb{X}_{1}}+I_{\mathbb{X}_{2}}\right) \rightarrow 0,
$$

we have $\operatorname{dim}_{\mathbb{k}}\left(I_{\mathbb{X}_{1}}+I_{\mathbb{X}_{2}}\right)_{5}=\left(\begin{array}{c}5+2 \\ 2\end{array}\right)$. By the same method as above, one can show that $\mathbb{X}$ has generic Hilbert function when $d=6,7$, and so

$$
\operatorname{dim}_{\mathbb{k}}\left(I_{\mathbb{X}_{1}}+I_{\mathbb{X}_{2}}\right)_{d}=\left(\begin{array}{c}
d+2 \\
2
\end{array}\right),
$$

for $5 \leq d \leq 7$, which completes the proof.

Theorem 2.2. Let $\lambda=(1, \ldots, 1,3)$ and let $\mathbb{X}$ be the union of two star-configurations $\mathbb{X}_{1}:=\mathbb{X}_{1}^{(\lambda)}$ and $\mathbb{X}_{2}:=\mathbb{X}_{2}^{(\lambda)}$ in $\mathbb{P}^{2}$ with $d \geq 8$. Then

$$
\operatorname{dim}_{\mathbb{K}}\left(I_{\mathbb{X}_{1}}+I_{\mathbb{X}_{2}}\right)_{d}=4 d+8 .
$$


Proof. First, we assume that $\mathbb{X}_{1}$ and $\mathbb{X}_{2}$ are defined by $L_{1}, \ldots, L_{d-3}, L_{d-2}^{3}$ and $M_{1}, \ldots, M_{d-3}, M_{d-2} M_{d-1}^{2}$, respectively, where $L_{i}$ and $M_{j}$ are linear forms in $R$ for every $i$ and $j$. Without loss of generality, we assume

$$
\begin{array}{lcl}
L_{1} & \text { vanishes on } d+1 \text { points } & P_{1,2}, \ldots, P_{1, d-3}, \mathcal{P}_{1, d-2}, \boldsymbol{Q}_{1, d-1}, \\
L_{2} & \text { vanishes on } d \text { points } & P_{2,3}, \ldots, P_{3, d-3}, \mathcal{P}_{2, d-2}, \boldsymbol{Q}_{2, d-1}, \\
& \vdots & \\
L_{d-3} & \text { vanishes on } 5 \text { points } & \mathcal{P}_{d-3, d-2}, \boldsymbol{Q}_{d-3, d-1} .
\end{array}
$$

By Bezóut's Theorem, for $N \in\left(I_{\mathbb{X}}\right)_{d}, N=L_{1} \cdots L_{d-3} N^{\prime}$ for some $N^{\prime} \in R_{3}$. Since a linear star-configuration $\mathbb{Y}$ in $\mathbb{P}^{2}$ defined by $M_{1}, \ldots, M_{d-2}$ has no generators in degree 3 and $N^{\prime}$ has to vanishes on all points in $\mathbb{Y}$, we see that $N^{\prime}=0$, i.e., $N=0$, and so $\operatorname{dim}_{\mathbb{k}}\left(I_{\mathbb{X}}\right)_{d}=0$.

Using the following exact sequence

$$
0 \rightarrow R / I_{\mathbb{X}} \rightarrow R / I_{\mathbb{X}_{1}} \oplus R / I_{\mathbb{X}_{2}} \rightarrow R /\left(I_{\mathbb{X}_{1}}+I_{\mathbb{X}_{2}}\right) \rightarrow 0,
$$

we have

$$
\operatorname{dim}_{\mathbb{k}}\left(I_{\mathbb{X}_{1}}+I_{\mathbb{X}_{2}}\right)_{d}=2 \cdot \operatorname{dim}_{\mathbb{k}} R_{d}-2 \cdot \operatorname{deg}\left(\mathbb{X}_{1}\right)=4 d+8,
$$

which completes the proof of this theorem.

Lemma 2.3. Let $\lambda=(1,3,3)$ or $(1,1,3,3)$, and $\mathbb{X}_{1}:=\mathbb{X}_{1}^{(\lambda)}$ and $\mathbb{X}_{2}:=$ $\mathbb{X}_{2}^{(\lambda)}$ be star-configurations in $\mathbb{P}^{2}$. Then $\mathbb{X}:=\mathbb{X}_{1} \cup \mathbb{X}_{2}$ has generic Hilbert function. In particular, $\operatorname{dim}_{\mathbb{k}}\left(I_{\mathbb{X}_{1}}+I_{\mathbb{X}_{2}}\right)_{d}=\left(\begin{array}{c}d+2 \\ 2\end{array}\right)$.

Proof. We shall introduce only the proof for the case $\lambda=(1,3,3)$, and we omit the proof for the case $\lambda=(1,1,3,3)$ since it simply reiterates the same arguments we will use. So we assume $\lambda=(1,3,3)$. Let $\lambda^{\prime}=(3,3)$, and $\mathbb{Y}_{1}:=\mathbb{X}_{1}^{\left(\lambda^{\prime}\right)}$ and $\mathbb{Y}_{2}:=\mathbb{X}_{2}^{\left(\lambda^{\prime}\right)}$ be star-configurations in $\mathbb{P}^{2}$. Let $\mathbb{Y}_{1}$ and $\mathbb{Y}_{2}$ be defined by $L_{2} L_{3} L_{4}, L_{5} L_{6} L_{7}$ and $M_{2} M_{3} M_{4}, M_{5} M_{6} M_{7}$, respectively, where $L_{i}$ and $M_{j}$ are linear forms in $R$ for every $i, j$. Then it is not hard to see that the Hilbert function of $\mathbb{Y}:=\mathbb{Y}_{1} \cup \mathbb{Y}_{2}$ is

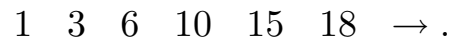

Now assume that $\mathbb{X}_{1}:=\mathbb{X}_{1}^{(\lambda)}$ is defined by $L_{1}, L_{2} L_{3} L_{4}, L_{5} L_{6} L_{7}$, where $L_{1}$ is a linear form in $R$, and $\mathbb{Z}:=\mathbb{X}_{1} \cup \mathbb{Y}_{2}$. Using the following exact sequence

$$
0 \rightarrow R / I_{\mathbb{Z}} \rightarrow R / I_{\mathbb{Y}} \oplus R /\left(L_{1}, G_{6}\right) \rightarrow R /\left(I_{\mathbb{Y}}, L_{1}, G_{6}\right) \rightarrow 0,
$$

where $G_{6}=L_{2} \cdots L_{7}$, we obtain the following Hilbert functions. 


$$
\begin{array}{rccccccccc}
\mathbf{H}\left(R / I_{\mathbb{Z}},-\right) & : & 1 & 3 & 6 & 10 & 15 & - & 24 & \rightarrow, \\
\mathbf{H}\left(R / I_{\mathbb{Y}},-\right) & : & 1 & 3 & 6 & 10 & 15 & 18 & 18 & \rightarrow, \\
\mathbf{H}\left(R /\left(L_{1}, G_{6}\right),-\right) & : & 1 & 2 & 3 & 4 & 5 & 6 & 6 & \rightarrow, \\
\mathbf{H}\left(R /\left(I_{\mathbb{Y}}, L_{1}, G_{6}\right),-\right) & : & 1 & 2 & 3 & 4 & 5 & - & 0 & \rightarrow, \\
\mathbf{H}\left(R /\left(I_{\mathbb{Y}}, L_{1}\right),-\right) & : & 1 & 2 & 3 & 4 & 5 & 3 & 0 & \rightarrow .
\end{array}
$$

By Bezóut's Theorem, it is easily to show that $\left(I_{\mathbb{Z}}\right)_{5}=\{0\}$, and so the

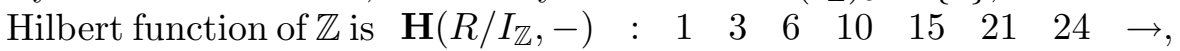
as we wished. Using the same idea as above and by Bezóut's Theorem, one can show that $\mathbb{X}$ has generic Hilbert function. Therefore, we get that

$$
\operatorname{dim}_{\mathbb{k}}\left(I_{\mathbb{X}_{1}}+I_{\mathbb{X}_{2}}\right)_{d}=\left(\begin{array}{c}
d+2 \\
2
\end{array}\right),
$$

for $d=7,8$, as we wished.

By the same idea as in the proof of Theorem 2.2, the following theorem can be easily obtained, and so we omit the proof.

Theorem 2.4. Let $\lambda=(\underbrace{1, \ldots, 1}_{(s-\ell) \text {-times }}, \underbrace{3, \ldots, 3}_{\ell \text {-times }})$ and let $\mathbb{X}$ be the union of two star-configurations $\mathbb{X}_{1}:=\mathbb{X}_{1}^{(\lambda)}$ and $\mathbb{X}_{2}:=\mathbb{X}_{2}^{(\lambda)}$ in $\mathbb{P}^{2}$ with either $\ell \geq 3$ or $\ell=2$ and $d \geq 9$. Then $\operatorname{dim}_{\mathbb{k}}\left(I_{\mathbb{X}_{1}}+I_{\mathbb{X}_{2}}\right)_{d}=4 d+6 \ell+2$.

\section{Varieties of reducible forms and their secants}

We first recall the definition of the secant variety $\operatorname{Sec}_{s-1}(\mathbb{X})$ to the variety $\mathbb{X}$ in $\mathbb{P}^{n}$. Let $\lambda \vdash d$ denote a partition of the integer $d$, i.e.

$$
\lambda=\left(d_{1}, \ldots, d_{s}\right) \text { where } 1 \leq d_{1} \leq \cdots \leq d_{s} \text { and } \sum_{i=1}^{s} d_{i}=d .
$$

We associate a variety, denoted by $\mathbb{X}_{\lambda, n}$, to $R=\mathbb{k}\left[x_{0}, x_{1}, \ldots, x_{n}\right]$ and $\lambda$, which is defined by

$$
\mathbb{X}_{\lambda, n}:=\left\{[F] \in \mathbb{P}\left(R_{d}\right) \mid F=F_{1} \cdots F_{s}, \operatorname{deg} F_{i}=d_{i}\right\} .
$$

Such varieties are called varieties of reducible forms. If $\lambda$ is the $d$-tuple $(1, \ldots, 1)$, then the variety is often referred to as the variety of completely decomposable forms or split forms. In this case, $\mathbb{X}_{\lambda, n}$ is denoted by $\operatorname{Split}_{d}\left(\mathbb{P}^{n}\right)$.

Since the map below has only finite fibers, $\mathbb{P}\left(R_{d_{1}}\right) \times \cdots \times \mathbb{P}\left(R_{d_{s}}\right) \longrightarrow \mathbb{X}_{\lambda, n}$, where $\left[F_{1}\right] \times \cdots \times\left[F_{s}\right] \longrightarrow\left[F_{1} \cdots F_{s}\right]$ 
the dimension of $\mathbb{X}_{\lambda, n}$ is

$$
\operatorname{dim} \mathbb{X}_{\lambda, n}=\left(\left(\begin{array}{c}
d_{1}+n \\
n
\end{array}\right)-1\right)+\cdots+\left(\left(\begin{array}{c}
d_{s}+n \\
n
\end{array}\right)-1\right)=\sum_{i=1}^{s}\left(\begin{array}{c}
d_{i}+n \\
n
\end{array}\right)-s .
$$

Definition 3.1. Let $\mathbb{X}_{1}, \ldots, \mathbb{X}_{s}$ all be non-degenerate, reduced and irreducible varieties in $\mathbb{P}^{n}$ with $\operatorname{dim} \mathbb{X}_{i}=d_{i}$.

(a) Choose points $P_{i} \in \mathbb{X}_{i}$ such that $\left\{P_{1}, \ldots, P_{s}\right\}$ are linearly independent (and so $s \leq n$ ). The join of $\left\{P_{1}, \ldots, P_{s}\right\}$ is the linear space spanned by the points, i.e.,

$$
\Lambda\left(P_{1}, \ldots, P_{s}\right):=\left\langle P_{1}, \ldots, P_{s}\right\rangle \simeq \mathbb{P}^{s-1} .
$$

(b) The join of $\mathbb{X}_{1}, \ldots, \mathbb{X}_{s}$ is $\Lambda\left(\mathbb{X}_{1}, \ldots, \mathbb{X}_{s}\right):=$ $\bar{U}\left\{\Lambda\left(P_{1}, \ldots, P_{s}\right) \mid\right.$ for $P_{1}, \ldots, P_{s}$ linearly independent, $\left.P_{i} \in \mathbb{X}_{i}\right\}$.

(c) If $\mathbb{X}_{1}=\cdots=\mathbb{X}_{s}=\mathbb{X}$ with $\operatorname{dim} \mathbb{X}=d$, then we write $\Lambda\left(\mathbb{X}_{1}, \ldots, \mathbb{X}_{s}\right)$ $=\operatorname{Sec}_{s-1}(\mathbb{X})$ and call it the $(s-1)$-st secant variety to $\mathbb{X}$.

The number of parameters shows that the upper bound of the dimension of the join is

$$
\operatorname{dim} \Lambda\left(\mathbb{X}_{1}, \ldots, \mathbb{X}_{s}\right) \leq \min \left\{n, \sum_{i=1}^{s} d_{i}+(s-1)\right\},
$$

and thus

$$
\operatorname{dim} \operatorname{Sec}_{s-1}(\mathbb{X}) \leq \min \{n, d s+(s-1)\} .
$$

Definition 3.2. Let $\mathbb{X} \subset \mathbb{P}^{n}$ be a projective variety of dimension $d$. Then the expected dimension of the secant variety $\operatorname{Sec}_{s-1}(\mathbb{X})$ to $\mathbb{X}$ is defined by

$$
\operatorname{expdim}\left(\operatorname{Sec}_{s-1}(\mathbb{X})\right)=\min \{n, d s+(s-1)\} .
$$

However, the expected dimension of $\operatorname{Sec}_{s-1}(\mathbb{X})$ is not always the same as $\operatorname{dim} \operatorname{Sec}_{s-1}(\mathbb{X})$. If $\operatorname{expdim}\left(\operatorname{Sec}_{s-1}(\mathbb{X})\right)-\operatorname{dim} \operatorname{Sec}_{s-1}(\mathbb{X})>0$, we say that the secant variety $\operatorname{Sec}_{s-1}(\mathbb{X})$ to $\mathbb{X}$ is defective.

Since we are interested in the secants to the varieties of reducible forms, we introduce another important result in [5] to find a description of the tangent space at a generic point of those varieties.

Proposition 3.3 ([5]). Let $\lambda \vdash d, \lambda=\left(d_{1}, \ldots, d_{s}\right)$ and let $\mathbb{X}_{\lambda, n} \subset$ $\mathbb{P}^{\left(\begin{array}{c}d+n \\ n\end{array}\right)-1}$. Let $P=\left[F_{1} \cdots F_{s}\right]$ be a generic point of $\mathbb{X}_{\lambda, n}$ where $\operatorname{deg} F_{i}=$ $d_{i}, i=1, \ldots, s$. Then $T_{P, \mathbb{X}_{\lambda, n}}=\mathbb{P}\left(V_{P}\right)$ where $V_{P}$ is the subspace of $R_{d}=\mathbb{k}\left[x_{0}, \ldots, x_{n}\right]_{d}$ defined by $V_{P}:=\left(\tilde{F}_{1}, \ldots, \tilde{F}_{s}\right)$, where $\tilde{F}_{i}=\frac{\prod_{j=1}^{s} F_{j}}{F_{i}}$ for every $i=1, \ldots, s$.

The following corollary is useful for finding whether or not the given secant varieties are defective. 
Corollary 3.4 ([5]). Let $\lambda \vdash d, \lambda=\left(d_{1}, \ldots, d_{s}\right)$ and let $\mathbb{X}_{\lambda, n} \subset$ $\mathbb{P}^{\left(\begin{array}{c}d+n \\ n\end{array}\right)-1}$. Let $P_{1}, \ldots, P_{s}$ be $s$ generic points on $\mathbb{X}_{\lambda, n}$. Then

$$
\operatorname{dim} \operatorname{Sec}_{s-1}\left(\mathbb{X}_{\lambda, n}\right)=\left[\left(\begin{array}{c}
d+n \\
n
\end{array}\right)-\mathbf{H}(A, d)\right]-1=\operatorname{dim}_{\mathbb{k}} I_{d}-1
$$

where $A=R / I$ and $I=\mathcal{T}_{P_{1}}+\cdots+\mathcal{T}_{P_{s}}$.

In this paper, we are interested in the secant variety $\operatorname{Sec}_{1}\left(\mathbb{X}_{\lambda, 2}\right)$ to the variety $\mathbb{X}_{\lambda, n}:=\left\{[F] \in \mathbb{P}\left(R_{d}\right) \mid F=F_{1} \cdots F_{s}\right.$, $\operatorname{deg} F_{i}=1$ or 2$\}$.

In $[3]$ and $[6]$ the authors showed that the secant variety $\operatorname{Sec}_{1}\left(\mathbb{X}_{\lambda, n}\right)=$ $\operatorname{Sec}_{1}\left(\operatorname{Split}_{d}\left(\mathbb{P}^{n}\right)\right)$ is not defective for $n \geq 2$. Moreover, since it is not hard to show that the secant variety $\operatorname{Sec}_{1}\left(\mathbb{X}_{\lambda, 2}\right)$ is not defective when $d_{i}=3$ for every $i$, we shall not introduce the proof in this paper. Thus we assume that $d_{1}=\cdots=d_{s-\ell}=1$ and $d_{s-\ell+1}=\cdots=d_{s}=3$ with $1 \leq \ell<s$ for the rest of this paper. We now introduce the main theorem in this paper.

Theorem 3.5. Let $\lambda \mapsto d$ and $\lambda=(\underbrace{1, \ldots, 1}_{(s-\ell) \text {-times }}, \underbrace{3, \ldots, 3}_{\ell \text {-times }})$. Then the secant variety $\operatorname{Sec}_{1}\left(\mathbb{X}_{\lambda, 2}\right)$ is not defective for $s \geq 3$ and $1 \leq \ell<s$.

Proof. If $d=5$ and $\ell=1$, then by Lemma 2.1 and Corollary 3.4,

$\operatorname{expdimSec}{ }_{1}\left(\mathbb{X}_{\lambda, 2}\right)$

$=\min \left\{2 \cdot \operatorname{dim}\left(\left(\mathbb{P}\left(R_{1}\right) \times \mathbb{P}\left(R_{1}\right) \times \mathbb{P}\left(R_{1}\right) \times \mathbb{P}\left(R_{3}\right)\right)+1,\left(\begin{array}{c}5+2 \\ 2\end{array}\right)-1\right\}\right.$

$=20=\operatorname{dim}_{\mathbb{k}}\left(I_{\mathbb{X}_{1}}+I_{\mathbb{X}_{2}}\right)_{5}-1=\operatorname{dim}_{\operatorname{Sec}_{1}}\left(\mathbb{X}_{\lambda, 2}\right)$.

By the same method as above with Lemmas 2.1, 2.3, and Corollary 3.4, one can see that $\operatorname{expdimSec}\left(\mathbb{X}_{\lambda, 2}\right)=\operatorname{dim} \operatorname{Sec}_{1}\left(\mathbb{X}_{\lambda, 2}\right)$ for either $d=6,7$ and $\ell=1$ or $d=7,8$ and $\ell=2$.

Now suppose either $\ell=1$ and $d \geq 8$ or $\ell=2$ and $d \geq 9$. Then, by Theorems 2.2, 2.4, and Corollary 3.4,

$$
\begin{aligned}
& \operatorname{expdimSec}\left(\mathbb{X}_{\lambda, 2}\right) \\
& =\min \left\{2 \cdot \operatorname{dim}\left((\underbrace{\mathbb{P}\left(R_{1}\right) \times \cdots \times \mathbb{P}\left(R_{1}\right)}_{(s-1) \text {-times }} \times \mathbb{P}\left(R_{3}\right))+1,\left(\begin{array}{c}
d+2 \\
2
\end{array}\right)-1\right\}\right. \\
& =4 d+7(\text { since } d \geq 8) \\
& =\operatorname{dim}_{\mathbb{K}}\left(I_{\mathbb{X}_{1}}+I_{\mathbb{X}_{2}}\right)_{d}-1=\operatorname{dim} \operatorname{Sec}_{1}\left(\mathbb{X}_{\lambda, 2}\right),
\end{aligned}
$$

and

$$
\begin{aligned}
& \operatorname{expdimSec}_{1}\left(\mathbb{X}_{\lambda, 2}\right) \\
& =\min \left\{2 \cdot \operatorname{dim}(\underbrace{\mathbb{P}\left(R_{1}\right) \times \cdots \times \mathbb{P}\left(R_{1}\right)}_{(s-2) \text {-times }} \times \mathbb{P}\left(R_{3}\right) \times \mathbb{P}\left(R_{3}\right))+1,\left(\begin{array}{c}
d+2 \\
2
\end{array}\right)-1\right\} \\
& =4 d+13(\text { since } d \geq 8)=\operatorname{dim}_{\mathbb{k}}\left(I_{\mathbb{X}_{1}}+I_{\mathbb{X}_{2}}\right)_{d}-1=\operatorname{dim~Sec}_{1}\left(\mathbb{X}_{\lambda, 2}\right),
\end{aligned}
$$


respectively, as we wished.

Now assume that $\ell \geq 3$. Then by Theorem 2.4 and Corollary 3.4,

$$
\begin{aligned}
& \text { expdimSec } 1\left(\mathbb{X}_{\lambda, 2}\right) \\
& =\min \left\{2 \cdot\left((\underbrace{\mathbb{P}\left(R_{1}\right) \times \cdots \times \mathbb{P}\left(R_{1}\right)}_{(s-\ell) \text {-times }} \times \underbrace{\mathbb{P}\left(R_{3}\right) \times \cdots \times \mathbb{P}\left(R_{3}\right)}_{\ell \text {-times }})+1,\left(\begin{array}{c}
d+2 \\
2
\end{array}\right)-1\right\}\right. \\
& =4 d+6 \ell+1(\text { since } d \geq 3 \ell+1) \\
& =\operatorname{dim}_{\mathbb{k}}\left(I_{\mathbb{X}_{1}}+I_{\mathbb{X}_{2}}\right)_{d}-1 \\
& =\operatorname{dim} \operatorname{Sec}_{1}\left(\mathbb{X}_{\lambda, 2}\right),
\end{aligned}
$$

which completes the proof.

Now we give a question on secant varieties to the variety $\mathbb{X}_{\lambda, n}$.

QUeSTION 3.6. Is the secant variety $\operatorname{Sec}_{s-1}\left(\mathbb{X}_{\lambda, 2}\right)$ to the variety $\mathbb{X}_{\lambda, 2}$ non-defective for $s>2$ when $\lambda=(1, \ldots, 1,3, \ldots, 3)$ ?

\section{References}

[1] J. Ahn and Y. S. Shin, The Minimal Free Resolution of A Star-Configuration in $\mathbb{P}^{n}$ and The Weak-Lefschetz Property, J. Korean of Math. Soc. 49 (2012), no. 2, 405-417.

[2] J. Ahn and Y. S. Shin, The Minimal Free Resolution of a Fat Star-configuration in $\mathbb{P}^{n}$, Algebra Colloquium, To appear.

[3] E. Arrondo and A. Bernardi, On the variety parameterizing completely decomposable polynomials, J. Pure Appl. Algebra 215 (2011), no. 3, 201-220.

[4] J. Alexander and A. Hirschowitz, Polynomial interpolation in several variables, J. Algebraic Geom. 4 (1995), no. 2, 201-222.

[5] E. Carlini, L. Chiantini, and A. V. Geramita, Complete intersections on general hypersurfaces, Mich. Math. J. 57 (2008), 121-136.

[6] Y. S. Shin, Secants to The Variety of Completely Reducible Forms and The Union of Star-Configurations, Journal of Algebra and its Applications, 11 (2012), no. 6, 1250109 (27 pages).

[7] Y. S. Shin, Star-Configurations in $\mathbb{P}^{2}$ Having Generic Hilbert Functions and The Weak-Lefschetz Property, Comm. in Algebra 40 (2012), 2226-2242.

Department of Mathematics

Sungshin Women's University

Seoul 136-742, Republic of Korea

E-mail: ysshin@sungshin.ac.kr 